\title{
EVALUACIÓN DE LOS INTERESES PARTICIPATIVOS Y NECESIDADES FORMATIVAS DE PROFESORES Y PADRES EN LOS CENTROS ESCOLARES DE EDUCACIÓN PRIMARIA
}

\author{
EVALUATION OF PARTICIPATING INTERESTS AND TRAINING \\ NECESSITIES OF PROFESSORS AND PARENTS IN THE SCHOLASTIC \\ CENTERS OF PRIMARY EDUCATION
}

Rosario Ordóñez Sierra*

Universidad de Sevilla

\section{RESUMEN}

En este artículo damos a conocer los ámbitos escolares en los que familia y profesorado tienen interés por participar. Analizamos el tipo de ayuda que solicita el profesorado a los padres y las tareas y actividades en las que los padres están interesados en involucrarse. Recogemos información sobre las necesidades formativas que presentan padres y profesores para poder afrontar las exigencias participativas; los medios y profesionales más adecuados para que los formen.

Nuestro estudio se ha basado en un método de investigación mediante encuesta, participando 24 centros de Educación Primaria en Sevilla capital.

Palabras clave: Intereses, participación, necesidades formativas, padres, profesores, Educación Primaria.

\begin{abstract}
In this article we present the scopes scholastic in which family and teaching staff have interest to participate. We analyzed the type of aid that asks for the teaching staff to the parents and the tasks and activities in which the parents are interested in becoming jumbled. We collect on the training necessities that they present parents and professors to be able to confront the participative exigencies; the suitable means and professionals more so that they form them. Our study has been based on
\end{abstract}

\footnotetext{
* Es profesora en la Facultad de CC. de la Educación, Departamento de Didáctica y Organización Educativa, su tesis doctoral versó sobre Perspectivas sobre la participación de padres y profesorado en los centros de Educación Primaria de Sevilla. Pertenece al grupo de investigación competitivo: ISIS, dirigido por la Catedrática Carmen García Pastor, investigando principalmente en el campo de la integración. Correo-E: rordonez@us.es
} 
a method of investigation by means of survey, participating 24 centers of Primary Education in capital Seville.

Key words: Interests, participation, training necessities, parents, professors, Primary Education.

\section{Introducción}

En este artículo, en primer lugar, daremos a conocer los ámbitos escolares en los que familia y profesorado están interesados en participar; mostraremos el tipo de ayuda que solicita el profesorado a los padres y, las tareas y actividades en las que los padres están interesados en participar.

De otro lado, hemos detectado las necesidades formativas de familia y profesorado para el desarrollo de la participación, recogiendo información sobre las necesidades formativas que presentan padres y profesores para poder afrontar las exigencias participativas, así como los medios y los profesionales más adecuados para que los formen. Igualmente se han considerado las actividades formativas que realiza el profesorado de cara a la participación.

Martínez González (1999), expone que cada vez más, los padres y madres pertenecientes a sociedades cultural y económicamente desarrolladas demandan formación para educar correctamente a sus hijos/as. En nuestro país, esta necesidad de formación parte fundamentalmente de la actual realidad de la sociedad española. Los grandes cambios sociales acaecidos en los últimos años han conducido a modificaciones en las estructuras y en las dinámicas que las familias necesitan desarrollar para que tanto los padres y madres como los/as hijos/as puedan adaptarse a las nuevas exigencias sociales. Estos cambios han propiciado un proceso de nuclearización de la familia, de individualización de la sociedad, de diferenciación de roles y especializaciones, y de distancia geográfica entre generaciones. En nuestro país numerosas investigaciones han constatado la demanda y la necesidad que tienen los padres y las madres de formación específica en diversos campos de actuación (Pérez Delgado, 1994; Millán, 1996; Merino Rodríguez, 1997).

Veremos en el análisis de los resultados, cómo algunos padres reseñan que necesitan vías y procedimientos de formación con el fin de ser preparados para saber participar. Cuando unos padres afrontan la paternidad se plantean el nombre, cómo les gustaría que fuese su hijo, qué valores les van a inculcar, a qué escuela asistirá... pero cómo debe ser su comportamiento ante la educación de sus hijos va surgiendo sobre la marcha y en ese proceso surgen muchas dudas. Y ante la pregunta que se formulan muchos padres "qué hacer para..." una de las posibles soluciones que nosotros planteamos es participar en una Escuela Activa de Padres o en la Asociación de Madres y Padres, pudiendo ser éstos buenos mecanismos para dar respuesta a las necesidades de las familias. Con el paso del tiempo y la suma de experiencias se ha ido viendo que la cooperación entre padres y profesores, ya que ambos son conscientes de que les incumbe una parte básica en la responsabilidad del progreso normal del niño, es un camino para dar respuesta a los problemas.

La comunicación continuada y comprensiva entre padres y educadores es fundamental en todo el proceso educativo. Los padres tienen el derecho de conocer la marcha del proceso en relación con sus hijos y los educadores deben tener presente esta relación en todo momento. Es imprescindible que familia y escuela funcionen de una forma coordinada, aunando objetivos y multiplicando esfuerzos (Moreno, 2001). 
Numerosos estudios han mostrado que a través de la relación entre familia y escuela el alumnado consigue mejorar su rendimiento académico, por ejemplo estudios realizados en Estados Unidos (Ford, 1998; Potter, 2000; Ramírez, 2000), o los realizados en Canadá (Deslandes y Rover, 1997) y en Alaska (Sandham, 1998). Igualmente otros estudios explicitan que no sólo se consigue mejorar el rendimiento académico a través de la actuación sobre la implicación familiar, sino que también se desarrollan actitudes y comportamientos positivos que enriquecen al alumnado (Henderson, 1989; Balster, 1991; Martínez González 1996; OCDE, 1997; Molero, 2003).

Molero en 2003 realizaba igualmente un estudio en el que analizaba la valoración que hace el alumnado de la implicación de sus familias en su vida escolar y las actuaciones que los padres y madres llevan a cabo para implicarse en la educación de sus hijos e hijas; coincidimos en que la participación de todos los miembros de la comunidad educativa de los centros es importante, pero más aún la de los padres ya que son los auténticos responsables de la educación de sus hijos. El entendimiento, acercamiento y colaboración entre familia y escuela es una forma de enriquecer y favorecer el proceso de aprendizaje del alumnado; aunando los esfuerzos se favorecerá la consecución de una meta común: la formación integral del alumnado (Martínez González, 2000; Molero, 2003).

Por todo ello, en este artículo presentamos los resultados obtenidos a partir de los cuestionarios administrados a profesores y padres. Pretendiendo poner de manifiesto las opiniones sobre el apoyo que ofrecen los padres al profesorado, el tipo de apoyo que solicita el profesorado a los padres: económico, ayuda en actividades, planificación de la tarea docente, etc., y el interés de los padres por participar.

\section{Método}

\section{Población}

El profesorado objeto de estudio de esta investigación, han sido los profesores que impartían docencia en el nivel de Educación Primaria, en centros públicos y concertados; incluyendo a los generalistas y especialistas, así como los padres de alumnos de dichos niveles educativos. Para la selección de la muestra nos basamos en Downie y Heat (1981), utilizando un procedimiento de muestreo polietápico aleatorio estratificado y por conglomerados, utilizando como primer criterio los seis distritos municipales de Sevilla capital: Casco Antiguo, Macarena, Nervión, Este, Sur y Triana. Y, como segundo criterio de selección, hemos empleado la titularidad de los centros: Públicos y Concertados. La muestra se ha extraído de forma aleatoria en cada estrato utilizando una tabla de números al azar.

\section{Recogida de información}

La información objeto de análisis que presentamos es la obtenida por medio de los cuestionarios sobre participación escolar que fueron dirigidos a profesores y padres, con la intención de conocer el grado de participación en los centros escolares de Educación Primaria en Sevilla capital. 
El proceso de elaboración y validación del cuestionario aplicado constó de diferentes fases. En primer lugar, una vez que estuvo elaborado el cuestionario, dicha versión inicial, se denominó versión $\mathrm{n}^{\mathrm{o}} 0$, a la cual se le realizó un estudio piloto dirigido a determinar la adecuación del contenido y la forma de las cuestiones a la población destinataria. Para ello se implicó en la aplicación piloto a sujetos similares a aquellos a los que iban a ser dirigidos posteriormente dichos cuestionarios. Tuvimos en cuenta todas las aportaciones y dudas acerca de la adecuación de los ítems a los objetivos, dificultad o ambigüedad de los términos empleados, formato, presentación, extensión, etc. Atendiendo a las sugerencias recibidas se modificó la versión inicial (versión $\left.n^{0} 0\right)$.

Después de dicha aplicación piloto, se perfiló la primera versión del cuestionario. Decidiéndose posteriormente, realizar una validación de contenido, basada en el juicio de expertos. Para ello se elaboró un protocolo anexo a la encuesta en el que se pedía a los diferentes expertos que asignaran una puntuación de menor a mayor valor (del 1 al 5) en la medida que los ítems se adecuaran o no al propósito establecido, valorando la claridad de los ítems, la adecuación del lenguaje a las características de la población a la que se les iba a pasar el cuestionario, formato, presentación, claridad de instrucciones... y, pidiéndoles también todas las observaciones y sugerencias que estimasen oportunas.

\section{Resultados}

La muestra participante está compuesta por 24 centros de Primaria de la Capital de Sevilla, de los cuales 14 son públicos y 10 son concertados; involucrándose un total de 400 profesores y 1370 padres para ser encuestados, de los 24 centros públicos y concertados distribuidos entre los seis distritos municipales de Sevilla capital. Nuestro estudio se ha basado en buena medida en un método de investigación mediante encuesta. Tras administrar los cuestionarios utilizados, hemos obtenido respuestas de un total 186 profesores y 725 padres, lo que supone un $46,5 \%$ de respuesta entre los profesores y, un 52,92\% entre los padres. Consideramos, por tanto, que se trata de una muestra significativamente representativa.

Recordemos, que la muestra de profesores estaba compuesta por 186 docentes. En cuanto al sexo, 43 de ellos son hombres, lo que supone un $24,6 \%$ y 132 son mujeres $(75,4 \%)$ lo que viene a confirmar una vez más un predominio femenino (Torres Santomé, 1991; Rovira y Tomé, 1993; UNESCO, 1998) entre los profesores de niveles básicos en el sistema educativo; un 5,9\% de la muestra no ha respondido a este ítem.

Las edades de los docentes que han contestado el cuestionario oscila mayoritariamente entre los 46 y 55 años (40,8\%), seguido del intervalo de edades entre 36 y 45 años con un $21,2 \%$, y por último, un $17,4 \%$ de la muestra se sitúa entre los 26 y 35 años.

Al indagar sobre la situación administrativa de los profesores comprobamos que un elevado porcentaje de la muestra son propietarios definitivos de su plaza $(70,6 \%)$; sólo un $4,4 \%$ son propietarios provisionales y un $8,1 \%$ interinos.

Asimismo los cargos desempeñados por dichos docentes en la gestión y gobierno de sus respectivos centros, son los siguientes: Director/a: 9 (4,8\%); Secretario/a: 4 (2,2\%); Jefe/a de Estudios: 9 (4,8\%); Coordinador/a de equipo: 32 (17,2\%); Tutor/a: 112 (60,2\%); Ninguno: $31(16,7 \%)$. 
$\mathrm{Al}$ observar los datos anteriormente expuestos podemos destacar que 112 profesores son tutores de grupo $(60,2 \%)$ y que asimismo 32 son coordinadores de grupo $(17,2 \%)$, lo que nos puede hacer ver que gran parte del profesorado de esta muestra colabora en equipo con el resto de los profesores de su ciclo.

\section{Intereses participativos de los padres a juicio del profesorado}

\section{Relaciones entre profesorado y padres}

Los principales temas tratados por los padres cuando se entrevistan con los profesorestutores de sus hijos, son los que hacen referencia a la evolución en el proceso educativo de su hijo $(92,5 \%)$, la conducta manifestada por el hijo en su aula y/o centro educativo $(89,8 \%)$, intercambio de información referente a las características personales de su hijo $(82,8 \%)$ y las relaciones de su hijo con sus compañeros y/o conducta manifestada por el mismo tanto en la clase como en el centro (57,0\%). Algunos padres responden a la opción "otros" manifestando los siguientes temas tratados en las entrevistas:

a) Excursiones, fiestas, terrario, actividades escolares y extraescolares;

b) todos los que se crean importantes para la evolución del alumno;

c) actitudes y aptitudes del alumno;

d) responsabilidad.

El apoyo más solicitado por parte de los profesores a los padres es la ayuda en la realización (puesta en práctica) de actividades extraescolares $(51,1 \%)$, la opción referente a la organización por parte de los padres de dichas actividades presenta un índice de solicitud mucho menor (30,6\%). Esencialmente los profesores solicitan apoyo en la organización de fiestas $(48,9 \%)$ y ayuda para la realización de determinado tipo de tareas académicas $(44,6 \%)$, cuando éstas se relacionan con tareas pedagógicas y tareas más específicas dentro del campo de intervención del docente, como pueden ser por ejemplo el desarrollo de unidades didácticas o ayuda en la gestión del centro, sólo un 4,8\% y un 4,3\% respectivamente de profesores requiere la presencia y ayuda de padres.

\section{Participación de los padres en los centros educativos}

Estimábamos necesario en nuestro estudio, preguntar directamente al profesor sobre sus creencias, opiniones ante el interés real de los padres por participar en los centros y, en caso afirmativo interpelarles sobre cuáles consideraban que serían los aspectos o ámbitos en los que los padres se encontrarían más interesados por participar.

Para comenzar, es significativo el alto porcentaje asignado por parte de los profesores a la opción "a veces" (77,3\%). Por lo que los docentes estiman que los padres sólo se muestran interesados por participar en determinados momentos y/o aspectos. De ellos los más seleccionados son los siguientes:

a) Organización de fiestas $(45,2 \%)$;

b) pertenecer al AMPA $(38,7 \%)$;

c) formar parte del Consejo Escolar (35,5\%); 
d) organización de excursiones $(29,6 \%)$;

e) diseño y organización de actividades extraescolares $(24,2 \%)$;

f) realización de actividades en clase (7,5\%).

En la opción "otros", los profesores han añadido: Formación de sus hijos; dar charlas formativas, acompañar en actividades extraescolares...; en temas que ayuden en la formación de sus hijos; catequesis; ayudar al desarrollo de sus hijos; a veces a nivel individual, directamente lo que afecte a sus hijos.

\section{Intereses participativos desde la perspectiva de los padres}

Este apartado presenta los resultados alcanzados tras el análisis de las cuestiones administradas a padres, en aspectos tales como el interés de los padres por colaborar, los motivos por los que no colaboran aun considerando importante la relación familia-escuela y, las actividades extraescolares en las que participan.

Anteriormente hemos comentado las opiniones de los padres sobre la importancia de la participación en los centros escolares y el por qué. En este ítem les interpelamos directamente sobre si ellos están interesados en participar, respondiendo afirmativamente a dicha cuestión un $80,9 \%$ de la muestra.

Hemos pedido a los padres su opinión sobre la necesidad de la colaboración familia-escuela y, que manifestaran los motivos que pueden llevarles a no participar en el centro. Se les presentó una relación de opciones para que seleccionaran aquélla/s que se acercaran o coincidieran más con su situación. Del mismo modo, ante la inexistencia de opciones acorde con su postura, les dejamos una vez más la posibilidad abierta de la opción "otros" para explicitar mejor sus motivos. Es significativo, y por tanto lo hemos de reseñar, que 119 padres hacen uso de esta posibilidad, posteriormente mostraremos las respuestas presentadas.

En primer lugar reseñar que un $56,8 \%$ de los padres estiman que es necesaria su participación en los centros, pero que ellos no lo hacen por falta de tiempo $(56,8 \%)$. El resto de las opciones obtienen porcentajes bajos, por ejemplo un $12,8 \%$ de los padres aducen no hacerlo porque no saben en qué pueden participar y un $7,3 \%$ porque consideran que el centro se organiza muy bien y no los necesita.

A continuación mostramos las respuestas presentadas por los padres en la opción otros:

- Un gran número de padres alegan que no participan en los centros escolares de sus hijos por la incompatibilidad entre el horario de sus puestos de trabajo y el horario escolar. Veamos algunos ejemplos: "El horario de trabajo no me lo permite"; "Motivos de horarios, totalmente incompatibles".

- Otros padres no poseen tiempo para participar en los centros educativos de sus hijos porque además han de ocuparse de algún familiar enfermo: "Tengo que cuidar a mi madre enferma"; "Trabajo de lunes a domingo, cuido de mis tres hijos y cuido de mi madre enferma con la que vivimos".

- Algunos padres alegan su no-participación porque no han recibido ninguna información por parte del centro y no sabían en qué y cómo hacerlo: "No sé en qué puedo participar porque nadie me comunica nada"; "El centro motiva poco. Oferta de participación poco variada". 
- Por último, explicitan motivos diversos como que poseen poca preparación, no lo encuentran necesario, confían en la profesionalidad del centro en cuanto a enseñanza, no tienen tiempo o porque tienen los hijos muy pequeños y el poco tiempo del que disponen se lo dedican a ellos.

Del mismo modo, les preguntamos a los padres si participaban en las actividades extraescolares organizadas por el centro de su hijo. De los resultados obtenidos, podemos destacar que son muy pocos padres los que participan en dichas actividades, ya que un $43,9 \%$ de los padres no lo hace "nunca" y un 39,8\% "pocas veces".

Respecto al tipo de actividades en las que colaboran los padres, básicamente colaboran en fiestas $(23,7 \%)$, excursiones $(22,2 \%)$ y con el AMPA $(12,3 \%)$. Éstas son las modalidades más señaladas por los encuestados.

Un 2,1\% de padres especifica su participación en otro tipo de actividades extraescolares: Catequesis y pastoral; Informática; colaboración en otras necesidades; asistencia a la tómbola; trabajos manuales (caseta feria, paso Semana Santa); organización de obras de teatros; informática e inglés...

\section{Necesidades formativas desde la perspectiva de los profesores}

En este apartado pretendemos hacer referencia al tipo de actividades de formación que realiza el profesorado, como los medios que consideran más adecuados para que se les enseñe a fomentar la participación. Por otro lado se les interpela sobre si creen que los padres están preparados para participar, si necesitan orientación sobre la educación de sus hijos y, sobre quién consideran que debe formarlos.

\section{Actividades de formación realizadas por los profesores}

En el cuestionario para profesores sobre participación se ha preguntado el tipo de actividades de formación que realizan. El mayor índice de respuesta lo obtiene la asistencia a cursos con un $83,3 \%$, un $39,8 \%$ de profesores se integra en grupos de trabajo y un $33,3 \%$ asiste a jornadas para formarse, actualizarse.

Diferentes estudios corroboran la necesidad de colaboración familia-escuela (Díez, 1982; AA.VV. 1987; Villalta, Tschorne, Torrente, 1989; Ballesteros, 1995; Gil Guil, 1995; Vila Mendiburu 1995, 1998), la necesidad de que los profesores impliquen a los padres de sus alumnos en la intervención de los centros escolares, pero también son muchos los estudios que indican que ni padres ni profesores son formados o preparados para saber asumir esa tarea (Fish, 1990; Musitu, 1994; Pulpillo, 1982; García, 1984; Santos Guerra, 1995). Del mismo modo sucede con los profesores encuestados en este estudio. Al preguntar sobre la necesidad de formarse un $86,0 \%$ opina que en su formación debería incluirse una preparación para saber fomentar la participación, indicando que los cursos serían la vía más aconsejable (46,8\%), seguidos de la realización de seminarios $(34,4 \%$ ) y un $19,9 \%$ estima la asistencia a conferencias como alternativa a la formación en dicho campo. Es importante reseñar que tan sólo un $15,6 \%$ del profesorado considera interesante o conveniente insertar en su formación inicial una asignatura que les prepare para saber fomentar la participación de ellos mismos en el centro, así como la de los padres de sus alumnos. 
Además de las respuestas comentadas, un 10,8\% de profesores contesta a esta pregunta señalando en la opción "otros", más medios o mecanismos para formarse:

- Algunos docentes proponen que se les imparta durante su formación inicial una o varias asignaturas que les prepare y capacite para abordar dicha tarea: "Incidir en este tema en toda la carrera"; "Parte de alguna asignatura en la formación".

- Otros estiman que se podrían hacer grupos de trabajo, charlas, trabajo con otros centros para completar o dar respuesta a sus necesidades, plantean como solución al problema una formación continuada: "Periódicos, puestas en común exponiendo la problemática, la solución encontrada por unos u otros"; "Grupos de trabajo, ponencias, charlas, puestas en común”.

- Algunos docentes estiman que debe ser el centro quien debe dar respuesta a sus necesidades formativas: "Ser motivados en el propio centro"; "Atender a las diferentes necesidades de cada profesor demandando sus gustos, necesidades, etc. tanto fuera del centro como dentro".

- Por último, hay un docente que opina que se debería hacer todo lo que fuera necesario para cubrir dichas carencias formativas y, otro justamente lo contrario: "Todo aquello que fuese necesario para fomentar la colaboración mutua"; "Creo que participamos tanto o más de lo que nos corresponde”.

\section{Formación de madres y padres}

Al valorar la formación de los padres, los profesores consideran que poseen poca formación para poder y saber participar $(61,8 \%)$, los porcentajes asignados a las opciones de respuestas "ninguno" y "mucho" nivel de formación $(1,2 \%$ y $3,0 \%)$ respectivamente, son significativamente bajos.

Para las respuestas negativas, se ha obtenido información adicional sobre quiénes serían, a juicio de los profesores, los encargados de formar a los padres para la participación en los centros. Los docentes piensan que debían ser unos especialistas invitados al centro $(41,4 \%)$ quienes se encarguen de formar a los padres, seguido de la propuesta de especialistas tales como los pedagogos $(21,5 \%)$, psicólogos $(18,8 \%)$ y asistentes sociales $(18,8 \%)$.

Igualmente como se ha venido haciendo con otros ítems, se ha facilitado la oportunidad de añadir otros especialistas que los encuestados consideraran pertinentes, y nosotros no hayamos reseñado. En este sentido, un 8,6\% de profesores consideran que esta función corresponde a: Médicos; Escuela de Padres; Escuelas específicas con personal especializado.

Se ha cuestionado también a los profesores sobre si piensan que los padres necesitan formación acerca de la educación de sus hijos. Se les ofreció una escala de respuesta de "ninguna" a "mucha", opinando un $72,4 \%$ del profesorado que los padres necesitan bastante formación sobre cómo orientar y educar a sus hijos y un 22,9\% que requieren "mucha formación", por lo que los valores "ninguna" y "poca formación" prácticamente no son seleccionados.

Los profesores estiman que esta formación deben ofrecerla los psicólogos $(50,5 \%)$ y/o pedagogos $(51,1 \%)$, en menor medida piensan que es labor del profesorado $(26,3 \%)$. 
Necesidades formativas desde la perspectiva de los padres

Al preguntar a los padres sobre si se encuentran preparados para participar, la respuesta ofrecida es similar a la del profesorado. Las opciones "nada" y "mucho" obtienen porcentajes muy bajos $(2,0 \%$ y $6,2 \%)$ y, las opciones "poco" y "bastante" son más recogidas y contempladas por los padres, con porcentajes de selección muy equiparados. Comparativamente, la opción "poco" con un 49,6\%, es menos seleccionada por los padres que por el profesorado $(61,8 \%)$ y, la opción "bastante" es más elegida por los padres $(42,1 \%)$ que lo era por los profesores $(33,9 \%)$. Por lo que se deduce a través de estas cifras que el profesorado otorga menos formación a los padres que la que se asignan ellos mismos, aunque los padres reconozcan sus necesidades formativas.

Las respuestas de los padres a la cuestión sobre quiénes deberían ayudarles en su preparación viene a completar la información obtenida sobre este tema. Los padres, en contradicción con lo que pensaba el profesorado, consideran que corresponde a los docentes del centro $(53,4 \%)$ formarlos o prepararlos para participar. A éstos sigue la opción "especialistas, según el tema de que se trate, 47,2\%" como expertos para ayudarles en su preparación, por último, también destaca la posibilidad de encargar esta preparación a los psicólogos y pedagogos $(27,2 \%)$.

En la opción "otros", los padres hacen mención de las siguientes propuestas para ser formados:

En primer lugar, los padres estiman que ellos mismos pueden formarse o prepararse para participar: "Todo padre puede aportar algo aunque no tenga preparación"; "Los propios padres de cada hijo”.

Otro sector de padres considera que debe ser el centro quien debe adquirir la responsabilidad de prepararlos para saber participar en los centros educativos de sus hijos: "Responsables del centro"; "Los profesores son los que mejor conocen los problemas del centro".

Otras propuestas para que asuman dicha tarea son la administración, Escuela de Padres y personas versadas en el tema a tratar, aunque no tengan por qué ser especialistas.

En relación a la orientación que han requerido los padres para la educación de los hijos, los resultados indican que un $31,5 \%$ de los padres reconocen haberla necesitado. Al responder sobre quiénes deberían proporcionar dicha orientación, estiman una vez más que los profesores de los centros de sus hijos son los profesionales más adecuados para dar respuesta a esa necesidad (52,0\%), aunque siguen contemplando la posibilidad de que les puedan orientar psicólogos/pedagogos $(43,4 \%)$.

A los padres que respondieron afirmativamente, se les solicitó que especificaran los aspectos o temas sobre los que habían solicitado orientación, resultando las siguientes respuestas:

- Los padres han solicitado orientación e información sobre el comportamiento de sus hijos en las diferentes etapas educativas, con la intención de saber afrontar los problemas de sus hijos.

- Los padres han pedido que se les forme en todos aquellos aspectos que han considerado pertinente para saber afrontar la educación de sus hijos. 
- Otros padres han visitado al psicólogo para solicitarle orientación sobre la problemática de su hijo, unas veces con la intención de resolver dudas y otras con la de intentar resolver el problema que presentaba su hijo.

- Algunos padres han solicitado orientación a los docentes de sus hijos con la intención de conocer su marcha académica e intentar ayudarlos a superar sus problemas en caso de necesidad.

- Los padres en determinados momentos han solicitado orientación sobre técnicas de estudio, tipo apoyo que necesitan sus hijos, etc.

- Por último, encontramos que los padres explicitan temas muy diversos sobre los que han solicitado en algún momento orientación.

\section{Conclusiones}

El apoyo más reivindicado por parte de los profesores a los padres es la ayuda en la realización y organización de actividades extraescolares y de fiestas, son muy pocos los docentes que solicitan a los padres apoyo en el ámbito académico, ya que las repuestas referentes a la solicitud de ayuda en la gestión del centro o desarrollo de unidades didácticas obtienen porcentajes muy bajos. Cuando se entrevistan los padres y profesores-tutores principalmente hablan sobre la evolución en el proceso educativo y/o aula del niño e intercambian información sobre las características personales del niño.

La mayoría de los padres sí están interesados por participar, por ayudar en la educación de sus hijos y que generalmente ésta se centra en las actividades extraescolares. Cuando los padres participan en las actividades extraescolares de sus hijos que suelen ser pocas veces, básicamente colaboran en fiestas, excursiones o con el AMPA.

Las conclusiones generales de este apartado son las siguientes:

- Los profesores solicitan apoyo de los padres para la realización de actividades extraescolares, organización de fiestas. Muy pocos docentes solicitan ayuda en el ámbito académico.

- En las entrevistas entre padres y profesores se habla sobre la evolución del proceso educativo del niño e intercambian información sobre las características personales del mismo.

- La mayor parte de los docentes consideran que el interés real de los padres se reduce a un número determinado de aspectos, como la organización de fiestas, excursiones o pertenecer al AMPA o Consejo Escolar.

- Sin embargo, la mayoría de los padres manifiesta poseer interés real por participar, cuando no lo hacen es por falta de tiempo, información, formación o porque ya han participado otros años o el centro se organiza muy bien sin ellos.

- En realidad, se muestra una gran distancia entre el interés que, verbalmente muestran los padres y, la implicación real por lo que sucede en el colegio de sus hijos.

De otro lado, las actividades que los docentes ponen más en práctica para su formación continuada son la asistencia a cursos, jornadas o la formación de grupos de trabajo. Estimando que en su formación inicial se les debía preparar para saber participar y, expo- 
nen que la asistencia a seminarios, cursos y conferencias son una buena alternativa para formarse.

Cuando se trata de valorar la formación que poseen los padres para saber afrontar su participación en los centros educativos, los profesores estiman que poseen poca formación (Ordóñez Sierra, 1997). Especialistas invitados al centro, pedagogos y psicólogos son los profesionales propuestos por los docentes para formar a los padres para saber participar en los centros educativos. En relación, a lo expuesto anteriormente, los profesores se reafirman al aclarar que los padres tampoco están formados para saber orientar y educar a sus hijos e insisten en que dicha formación no corresponde ofrecerla a ellos, sino a especialistas como pedagogos o psicólogos.

La respuesta ofrecida por los padres acerca de su preparación para saber participar es similar a la ofrecida por el profesorado, aunque algo menos pesimista. Y sí, contradicen las respuestas manifestadas por los docentes sobre quiénes debía formarlos, porque los padres creen que deben ser los profesores de sus hijos quienes deben asumir dicha tarea, seguidos de los pedagogos y psicólogos.

\section{Las conclusiones generales quedan resumidas del siguiente modo:}

- Para formarse los docentes asisten a cursos, jornadas o forman grupos de trabajo.

- Los profesores estiman que los padres están pocos formados para participar en los centros educativos y para orientar y educar a sus hijos.

- Los profesores proponen que especialistas invitados al centro, pedagogos o psicólogos son los profesionales más adecuados para formar a los padres.

- Unos padres, consideran que se encuentran poco preparados para saber participar (contextos socioculturales bajos) y, otros bastante (centros concertados). Ambos reconocen necesitar orientación para educar a sus hijos y, consideran que los profesores son los profesionales que deben formarlos, seguidos de psicólogos o pedagogos.

En primer lugar, hemos de hacer constar que las implicaciones de este trabajo son diversas, ya que interesa seguir indagando y trabajando en el amplio y complejo campo de la participación.

Una vez más, se constata la diferencia existente entre lo que debiera ser y lo que es en realidad, los que dicen profesores y padres y lo que realmente hacen. Estableciéndose así, una vez más, la diferencia entre la teoría y la práctica, por lo que habría que insistir en superar esa línea separadora. Parece que no basta con crear estructuras de participación, sino que además es necesario formar a las personas para llegar a dar contenido y vida a las mismas y dinamizar los procesos sociales.

Para ello, desde nuestro punto de vista, sería importante abogar por un plan de Formación tanto para profesores como padres. Ambos sectores han dejado claro, en la presente investigación sus carencias formativas para saber afrontar adecuadamente las exigencias requeridas para la colaboración y participación mutua de profesores y padres en los centros educativos.

Hay que insistir en la formación de los padres, retomando la idea recogida en el Plan de Formación Nacional dirigido a padres o, potenciando las estructuras actuales, por ejemplo en Educación de Adultos o las Escuelas de Padres. Otra línea, a tener en cuenta, sería la implicación de otras instituciones, utilizando sus talleres, aulas de cultura, etc. 
Con respecto a la formación de profesores, creemos necesario plantear desde la formación inicial de profesores, ámbitos que desarrollen la cultura participativa. En la formación inicial se percibe un vacío importante en cuanto a su formación personal para implicarse en las estructuras participativas de sus centros y, de modo especial, para la interacción con las familias.

Dentro del marco de las implicaciones, consideramos que otra opción interesante sería realizar un estudio de caso, en el que se profundizara más en las ideas y opiniones de profesores y padres, realizando observaciones y entrevistas más profundas y exhaustivas. Animamos a próximas investigaciones estudiar la participación y necesidades de las familias con hijos con necesidades educativas especiales, estudiar la relación e interacción que se establece en los centros escolares donde están escolarizados sus hijos, tipo de necesidades, relaciones que se promueven con otros padres, los profesores-tutores, especialistas, etc.

\section{Limitaciones del estudio}

En el presente estudio hay que tener presente en primer lugar, que la muestra de padres es bastante amplia y que casi la totalidad de los mismos ha expuesto enriquecedores y diversidad de argumentos ante sus inquietudes y problemáticas sobre su participación en los centros escolares. Pero, cabría plantearse, ¿han cumplimentado el cuestionario sólo aquellos padres que tienen inquietudes y por tanto se refleja en sus respuestas? La misma pregunta se podría plantear a los docentes, recordemos que de 400 docentes sólo cumplimentaron el cuestionario 186 y de los 1370 padres 725 , si bien es cierto que son porcentajes elevados hay que recordar que muchos centros rechazaron directamente participar en la investigación, por lo que nos volvemos a cuestionar si los que han aceptado formar parte del estudio ¿son los que realmente participan, los que colaboran habitualmente en sus centros?, ¿están sesgados, en ese caso, los resultados? ... parece que no tendría por qué ser así, aunque la cuestión queda planteada.

Por ello, en futuros trabajos se podrían analizar las mismas dimensiones aumentando la población en los distintos contextos socioculturales para un mayor y mejor contraste de datos, a la vez que también consideramos interesante incluir en la muestra medios urbanos y rurales, con la intención de conocer cómo trabajan, se implican, intereses de padres y profesores de ambos medios.

\section{Referencias bibliográficas}

AA.VV. (1987). "Lo que los padres pueden hacer". Rev. Cuadernos de Pedagogía, no 147, 16-32.

Ballesteros Moscosio, M. A. (1995). "Necesidad de la participación de la familia en la escuela”. En LLorent Bedmar, V. Familia, Comunicación y Educación. Sevilla: KRONOS.

Balster, L. (1991). Involving at-risk families in their children's education. ERIC Clearing house of Educational Mangement: University of Oregon.

De los Ríos, R. (1986). "Los padres se inhiben del colegio de sus hijos". Rev. Comunidad Española, año XLVI, 17 de abril, 2-5.

Deslandes, R. y Rover, E. (1997). "Family Related Variables and Schools Disciplinary Events at Secondary Level”. Behavioral Disorders, 23 (1), 18-28. 
Díez, J. J. (1982). Familia-escuela una relación vital. Madrid: Narcea.

Fish, V. (1990a). "Introducing causality and power into family therapy theory: A correction to the systemic paradigm". Journal of Marital and Family Therapy, 1, 21-31.

Ford, M. y otros. (1998). "School-Family Partnerships: Children and Benedit". Teaching children mathematics, 4 (6), 310-312.

Gil Guil, M. (1995). "Interacción familia-escuela". En Llorent Bedmar, V. Familia, Comunicación y Educación. Sevilla: Kronos.

Herdenson, A. (1989). The evidence continues to grow. Parents involvement improves student achievement. Columbia, Maryland: National Committee for Citizens in Education.

Luengo, J. y Luzón, A. (2001). "El proceso de transformación de la familia tradicional y sus implicaciones educativas". Rev. Investigación en la escuela. Contexto familiar, contexto escolar, $\mathrm{n}^{\circ} 44$, $55-68$.

Martínez González, R. A. y Corral, N. (1996). "The need of partnership: A comparison of parents and children in Spain". Forum of Education, 1 (1), 73-83.

Martínez González, R. A. (1999). "Orientación educativa para la vida familiar”. Revista Española de Orientación y Psicopedagogía, vol. 10, n 17, primer semestre, 115-127.

Martínez González, R. A. y otros. (2000). "Dinamización de las relaciones familia-centro escolar a través de la formación del profesorado en este campo de actuación". Revista Española de Orientación y Psicopedagogía, 11 (9), 107-120.

Merino Rodríguez, C. et al. (1997). El taller de padres como recurso educativo. Granada: Ayuntamiento de Santa Fe.

Millán, M. (1996). Psicología de la familia. Valencia: Promolibro.

Molero, D. (2003). "Estudio sobre la implicación de las familias en el desarrollo de los procesos de enseñanza y aprendizaje”. Revista Española de Orientación y Psicopedagogía, 14 (1), primer semestre, 61-82.

Moreno, M. (2001). "Colaboración escuela-familias: análisis de una experiencia de escuela de padres en el I.E.S. Torreblanca de Sevilla durante el curso 1998/99”. Revista Española de Orientación y Psicopedagogía, vol. 12, n 22, segundo semestre, 341-353.

Musitu, G. y Allat, P. (1994). Psicosociología de la familia. Valencia: Albatros Educación.

OCDE (1997). Parents at partners in schooling. Paris: Centre for Educational Research and Innovation.

Ordóñez Sierra, R. (1997). Participación familia-escuela en los centros educativos: necesidades formativas. Trabajo de investigación dentro del programa de doctorado, dirigido por la doctora doña Isabel Ridao García. (inédito).

Pérez Delgado, E. (1994). Familia y Educación. Relaciones familiares y desarrollo personal de los hijos. Generalitat Valenciana.

Potter, C y otros (2000). Families, Schools, Communities: Learning Together School Volunteer Guide. Madison: Wisconsin State Dept. of Public Instruction.

Pulpillo Ruiz, A. J. (1982). La participación de los padres en la escuela. Madrid: Escuela Española.

Ramírez, A. Y. (2000). "High Teacher's View of Parent Involvement". American Secondary Education, 28 (4), 27-32.

Rovira, M. y Tomé, A. (1993). “La enseñanza: ¿una profesión femenina?”. Cuadernos para la coeducación, 4. Universitat de Barcelona. Institut de Ciéncies de L'Educació. Barcelona.

Sandham J. L. (1998). "Halfway Home”. Education Week, 18 (10), 36-41. 
Santos Guerra, M. A. (1995). "Democracia escolar o el problema de la nieve frita". En AA.VV.: Volver a pensar la educación. Prácticas y discursos educativos. Vol. I. Madrid: Morata, 128-141.

Torres Santomé, J. (1991). El currículum oculto. Madrid: Morata.

UNESCO (1998): Informe Mundial sobre la Educación. Los docentes y la enseñanza en un mundo en mutación. Madrid: Santillana/UNESCO.

Vila Mendiburu, I. (1995). "Relaciones familia-escuela". Rev. Cuadernos de Pedagogía, no 239, 14-16.

Vila Mendiburu, I. (1998). "Familia, escuela y comunidad”. Cuadernos de educación. Universidad de Barcelona: Horsori.

Villalta, M., Tschorne, P., Torrente, M. (1989). Los padres en la escuela. Barcelona: Laia.

Fecha de recepción: 12-06-06

Fecha de revisión: 29-04-08

Fecha de aceptación: 22-05-08 